\title{
Lusioersily
}

\section{Sampling and measurement methods for a study of childhood refractive error in a UK population}

O'Donoghue, L., Saunders, KJ., McClelland, JF., Logan, NS., Rudnicka, AR., Gilmartin, B., \& Owen, CG. (2010). Sampling and measurement methods for a study of childhood refractive error in a UK population. BRITISH JOURNAL OF OPHTHALMOLOGY, 94(9), 1150-1154. https://doi.org/10.1136/bjo.2009.167965

Link to publication record in Ulster University Research Portal

\author{
Published in: \\ BRITISH JOURNAL OF OPHTHALMOLOGY
}

Publication Status:

Published (in print/issue): 16/06/2010

DOI:

10.1136/bjo.2009.167965

\section{Document Version}

Publisher's PDF, also known as Version of record

\section{General rights}

Copyright for the publications made accessible via Ulster University's Research Portal is retained by the author(s) and / or other copyright owners and it is a condition of accessing these publications that users recognise and abide by the legal requirements associated with these rights.

\section{Take down policy}

The Research Portal is Ulster University's institutional repository that provides access to Ulster's research outputs. Every effort has been made to ensure that content in the Research Portal does not infringe any person's rights, or applicable UK laws. If you discover content in the Research Portal that you believe breaches copyright or violates any law, please contact pure-support@ulster.ac.uk. 


\section{BJO}

\section{Sampling and measurement methods for a study of childhood refractive error in a UK population}

L O'Donoghue, K J Saunders, J F McClelland, et al.

$\mathrm{Br} J$ Ophthalmol published online June 16, 2010

doi: 10.1136/bjo.2009.167965

Updated information and services can be found at:

http://bjo.bmj.com/content/early/2010/06/16/bjo.2009.167965.full.html

These include:

References This article cites 26 articles, 7 of which can be accessed free at: http://bjo.bmj.com/content/early/2010/06/16/bjo.2009.167965.full.html\#ref-list-1

$\mathbf{P}<\mathbf{P} \quad$ Published online June 16, 2010 in advance of the print journal.

Email alerting Receive free email alerts when new articles cite this article. Sign up in the service box at the top right corner of the online article.

\section{Notes}

Advance online articles have been peer reviewed and accepted for publication but have not yet appeared in the paper journal (edited, typeset versions may be posted when available prior to final publication). Advance online articles are citable and establish publication priority; they are indexed by PubMed from initial publication. Citations to Advance online articles must include the digital object identifier (DOIs) and date of initial publication.

To order reprints of this article go to:

http://bjo.bmj.com/cgi/reprintform

To subscribe to British Journal of Ophthalmology go to:

http://bjo.bmj.com/subscriptions 


\title{
Sampling and measurement methods for a study of childhood refractive error in a UK population
}

\author{
L O'Donoghue, ${ }^{1}$ K J Saunders, ${ }^{2}$ J F McClelland, ${ }^{2}$ N S Logan, ${ }^{3}$ A R Rudnicka, ${ }^{4}$ \\ B Gilmartin, ${ }^{5}$ C G Owen ${ }^{6}$
}

${ }^{1}$ School of Biomedical Sciences, University of Ulster, Coleraine, UK

${ }^{2}$ University of Ulster, Coleraine, UK

${ }^{3}$ Aston University, Ophthalmic Research Group, Birmingham, UK

${ }^{4}$ St George's, University of London, London, UK

${ }^{5}$ Aston University, Ophthalmic Research Group, Birmingham, UK

${ }^{6}$ St George's, University of London, London, UK

\section{Correspondence to} Lisa O'Donoghue, School of Biomedical Sciences, University of Ulster, Cromore Road, Coleraine BT52 1SA, UK; l.odonoghue@ulster.ac.uk

Accepted 28 November 2009

\section{ABSTRACT}

Background There is a paucity of data describing the prevalence of childhood refractive error in the United Kingdom. The Northern Ireland Childhood Errors of Refraction study, along with its sister study the Aston Eye Study, are the first population-based surveys of children using both random cluster sampling and cycloplegic autorefraction to quantify levels of refractive error in the United Kingdom.

Methods Children aged 6-7 years and 12-13 years were recruited from a stratified random sample of primary and post-primary schools, representative of the population of Northern Ireland as a whole.

Measurements included assessment of visual acuity, oculomotor balance, ocular biometry and cycloplegic binocular open-field autorefraction. Questionnaires were used to identify putative risk factors for refractive error. Results $399(57 \%)$ of $6-7$ years and $669(60 \%)$ of 12-13 years participated. School participation rates did not vary statistically significantly with the size of the school, whether the school is urban or rural, or whether it is in a deprived/non-deprived area. The gender balance, ethnicity and type of schooling of participants are reflective of the Northern Ireland population.

Conclusions The study design, sample size and methodology will ensure accurate measures of the prevalence of refractive errors in the target population and will facilitate comparisons with other populationbased refractive data.

Although refractive errors are common in children, ${ }^{1}$ their importance as a public health issue has often been underestimated. However, the financial costs can be considerable. In Australia, the provision of spectacles, contact lenses and refractive surgery for myopia runs into hundreds of millions of dollars. ${ }^{2}$ The management of associated ocular morbidity such as the increased risk of amblyopia and strabismus associated with hyperopia ${ }^{3}$ and the pathological complications associated with myopia ${ }^{4}$ also have significant cost implications.

Uncorrected refractive error is the most common cause of visual impairment in school-aged children in both industrialised and developing countries ${ }^{5}$ and can dramatically affect the learning capability and educational potential of children. ${ }^{1}$ Vision 2020, a World Health Organization initiative to eliminate avoidable causes of blindness by 2020, has estimated that there are 153 million people worldwide with visual impairment (presenting acuity <6/18) due to uncorrected refractive errors (http://www.v2020.org/).

Numerous epidemiological studies have described an increase in the prevalence of myopia in young adolescent eyes, ${ }^{6}$ especially in Asia. ${ }^{7}$ However, the evidence for changing myopia prevalence in white populations is equivocal. ${ }^{2}$ The prevalence of myopia also varies between countries even when subjects of similar ethnicity are compared, ${ }^{8} 9$ suggesting that prevalence rates may be population specific.

The few studies that have been published on childhood refractive error in the United Kingdom have examined selective populations such as children attending community eye clinics ${ }^{10}$ or optometric practice ${ }^{11}$ or have estimated the prevalence of refractive error based on vision screening data. ${ }^{12}$ Sorsby's ${ }^{13}$ description of the distribution of ocular refraction was carried out on a non-random sample of school-children and the participation rate was low (30-40\%). More recently, the Avon Longitudinal Study of Parents and Children used noncycloplegic closed-field autorefraction to estimate the prevalence of refractive error. ${ }^{14} 15$ Because of the effects of active accommodation, use of habitual refractive status in children can substantially overestimate the prevalence of myopia and underestimate the prevalence of hyperopia. ${ }^{16}$ Cycloplegic refraction is considered essential in childhood epidemiological surveys of refractive error, with cyclopentolate hydrochloride frequently being utilised. ${ }^{1} 1718$ In some such studies, participation rates of $>90 \%{ }^{19}{ }^{20}$ have been reported, confirming that its use is not a major barrier to participation.

The Northern Ireland Childhood Errors of Refraction (NICER) study examines the burden of refractive error in school children in Northern Ireland where the population is mainly white. It aims to be representative of Northern Ireland as a whole, with an appropriate mix of children from urban/rural areas and deprived/non-deprived areas. Associations with age, sex, sociodemographic and lifestyle determinants, especially the effect of urbanisation, are determined. The NICER study is a sister study to the Aston Eye Study, which examines refractive error in multiethnic urban school children in Birmingham, England. ${ }^{21} 22$ The current report describes the methodology used by the NICER study and presents data on the participation rates and characteristics of participants.

\section{MATERIALS AND METHODS \\ Ethics}

Approval for the study was obtained from the University of Ulster's Research Ethics Committee. The research adhered to the principles of the Declaration of Helsinki. 


\section{Study area, sample identification and sample size}

The two age groups (6-7 years and $12-13$ years) were selected to facilitate comparisons with other studies of childhood refractive error. ${ }^{18}$ School-based testing was used as compulsory education for children in Northern Ireland from 4 to 16 years of age ensures the present study captured children representative of the Northern Irish population.

Sample size calculations were based on $3 \%$ prevalence of myopia with $1 \%$ SE for $6-7$-year-olds and $10 \%$ prevalence with $1.5 \%$ SE for 12-13-year-olds. A modest inflation of the sample size was made to allow for clustering within school (30\%) and an assumed a response rate of $65 \%$ at both ages. Therefore, a minimum of 585 six- to seven-year-old children and 800 twelve- to thirteen-year-old children were required.

Data on population density and deprivation (Multiple Deprivation Measure) available from government databases (http://www.nisra.gov.uk/) were used to broadly classify the schools into four strata of urban/rural and deprived/not deprived. Stratified random sampling of schools was performed to obtain representation of schools and children across these four strata from four local government districts (Derry, Limavady, Coleraine and Ballymena). We aimed to recruit four primary schools and four post-primary schools from each stratum. All 6-7-year-olds in a primary school and two or more classes of 12-13-year-old children in post-primary schools were invited to participate and this resulted in slightly higher numbers overall of children being invited and examined. A randomisation list was prepared in advance, and if a school was unable or refused to participate, it was replaced by the next school on the list from the same stratum.

\section{Recruitment}

Initially, the school principal was sent a letter outlining the study, followed up several days later by telephone when arrangements were made to visit the school to explain the study in detail and to identify a suitable time in the school calendar for data collection to take place. Children who were invited to participate in the study attended an information session in the school that explained the rationale behind the study and the study procedures in detail. Children were given a detailed information pack for their parents/guardians, and written informed consent was obtained from a parent or other responsible adult before the child's participation in the study.

\section{Study personnel}

All personnel involved in data collection were checked by the Criminal Records Bureau and were trained in the exact protocols and procedures required. In line with similar large-scale studies ${ }^{118}$ multiple examiners were used. The study coordinator (LOD), a qualified registered optometrist, carried out all initial contact with the schools and was present at all times during data collection.

\section{Examination procedures}

The children were tested within school premises during the school day. Verbal assent was obtained from the children aged 6-7 years, and written consent from the 12-13-year-old children. The following protocol was used:

- Focimetry of any current spectacles.

- Assessment of ethnicity by the study coordinator.

- Monocular distance visual acuities (unaided and presenting) were measured at a distance of at least $3 \mathrm{~m}$, using the logMAR chart on the Test Chart 2000, a Windows-based computerised test chart (http://www.thomson-software-solutions.com/ html/test_chart_2000.html) and the by-letter scoring system.
- Assessment of heterophoria/tropia at distance (at least $3 \mathrm{~m}$, using the smallest letter on the logMAR chart that could be seen clearly with each eye) and near $(33 \mathrm{~cm}$, using an appropriately sized fixation target on the Budgie Stick) using the cover/uncover test both unaided and with spectacles if worn.

- Completion of a child questionnaire by the 12-13-year-old children to identify risk factors for myopia, including amount of time spent on near work and level of physical activity. Support for the questionnaire completion was available from the study personnel if required.

- Cycloplegia of both eyes using one drop of cyclopentolate hydrochloride 1\% (Minims ${ }^{\circledR}$ single dose, Chauvin Pharmaceuticals, Romford, UK) after instillation of one drop of proxymetacaine hydrochloride $0.5 \%$ (Minims ${ }^{\circledR}$ single dose, Chauvin Pharmaceuticals).

- Height (in centimetres) using the Leicester Height Measure (SECA, Hamburg, Germany).

- Weight (in kilograms) using Tanita digital scales, model HD-327 (Tanita, Middlesex, UK).

- Digital photography of the optic disc of both eyes using the Nidek NM200 Fundus Camera (Nidek, Aichi, Japan).

- Ocular biometry. One examiner (LOD) used the Zeiss IOLMaster (Carl Zeiss Meditec, Oberkochen, Germany) to measure axial length, anterior chamber depth and corneal curvature. ${ }^{23} 24$ At least three measurements of axial length, five simultaneous anterior chamber depth measurements and three corneal curvature readings were taken. Only axial length measurements with a signal-to-noise ratio greater than two were considered valid for subsequent analysis.

- Distance autorefraction, with the subject fixating the centre of a Maltese Cross, was carried out using the binocular openfield autorefractor, the Shin-Nippon SRW-5000 (Shin-Nippon, Tokyo, Japan), ${ }^{25}$ at least 20 min after the instillation of the eye drops. The presence of dilated pupils that were nonresponsive to light and an amplitude of accommodation of less than two dioptres were used to confirm that cycloplegia had been achieved before autorefractor measurement. The representative value as determined by the instrument was used in subsequent analyses.

- Adult questionnaires. After examination had been completed, the child was given an information pack for their parents/ guardians. This pack included a thank-you letter, drug information sheet and a detailed questionnaire designed to elicit risk factors for myopia, including child's birth history, family history and lifestyle questions. The parents/guardians were asked to complete the questionnaire and to return it directly to the study coordinator in the Freepost envelope provided.

\section{Definitions}

In line with recommendations from the World Health Organization, 'uncorrected visual acuity' is used to refer to unaided vision and 'presenting visual acuity' is the visual acuity with distance spectacles if worn. ${ }^{26}$

The spherical equivalent refractive error (SER: sphere $+1 / 2$ cylinder) of the right eye was used to classify participants as myopic, hyperopic or emmetropic. To facilitate comparisons with previously published studies, myopia prevalence was described as $-0.50 \mathrm{DS}$ or more myopia. SER was classified as hyperopic when greater than or equal to $+0.50 \mathrm{DS}$, with moderate hyperopia defined as a SER +2.00DS or higher, and emmetropia defined as a SER higher than-0.50DS and $<+0.50 D^{1}{ }^{1}{ }^{18}$ 
Table 1 Parental consent and participation rates

\begin{tabular}{lclllll}
\hline $\begin{array}{l}\text { Age group } \\
\text { (years) }\end{array}$ & $\begin{array}{l}\text { Number } \\
\text { invited }\end{array}$ & $\begin{array}{l}\text { Parental consent (\% } \\
\text { (median)) }\end{array}$ & $\begin{array}{l}\text { IOR parental consent \% } \\
\text { individual schools }\end{array}$ & $\begin{array}{l}\text { Participation (\% } \\
\text { (median)) }\end{array}$ & $\begin{array}{l}\text { IOR participation \% individual } \\
\text { schools }\end{array}$ & $\begin{array}{l}\text { Number of participants } \\
\text { examined }\end{array}$ \\
\hline $6-7$ & 700 & $62(62)$ & $58-72$ & $57(57)$ & $52-69$ & 399 \\
$12-13$ & 1115 & $65(71)$ & $59-78$ & $60(67)$ & $53-77$ & 669 \\
\hline
\end{tabular}

IQR, interquartile range.

\section{Follow-up}

Subsequent to the examination, if there were any concerns about the eye health of a participant, the parents/guardians were contacted directly advising them of the necessity for the child to have a full eye examination. No major adverse reactions to the eye drops were reported.

Parents/guardians who failed to return the questionnaire were sent a second copy, together with a Freepost envelope in which to return it.

\section{Data entry, analysis and statistics}

Initial data entry was carried out using Excel 2003. The data were anonymised by using an individual code for each participant. A separate password-protected file was created to store the names of the participants, together with their unique identity code. The data were then transferred to the statistical package (Intercooled Stata 9.2 software), where error checking was carried out before analysis. $t$ Tests were used to compare parental consent rates between deprived/non-deprived schools and urban/rural schools, with linear regression used to describe the association between parental consent rate and school size.

\section{RESULTS}

\section{Response rates and characteristics of participants}

Data collection took place between May 2006 and April 2008. Of the 16 primary schools originally identified, 15 participated in the study (94\%). Thirteen out of the 15 post-primary schools originally identified participated in the study (87\%). Suitable replacement schools were identified and participated in the study.

Table 1 describes the consent and participation rates for each age group. Consent was not established for $14 \%$ of primary school children and $20 \%$ of post-primary school children because of non-return of the forms. $24 \%$ of primary school children returned the consent form with consent actively declined compared with $14 \%$ of post-primary school children.

Of the children for whom parental consent was received, 93\% of the 12-13-year-old children and $92 \%$ of the 6-7-year-old children participated in the study. The difference between the consent rate and the actual participation rate was due to nonattendance on the day of testing $(3.7 \%$ primary, $4.6 \%$ postprimary) or the child refusing to consent to the instillation of eye drops (4.6\% primary, $2.5 \%$ post-primary).

One child in each age group was excluded from analysis due to the presence of Down's syndrome. Refractive and biometric data collection was complete for $99.8 \%$ of $12-13$-year-old children and $98.7 \%$ of $6-7$-year-old children.

There was no statistically significant difference between the parental consent rate in schools in deprived versus non-deprived areas $(t=0.96, p=0.35)$ or between schools in urban versus rural areas $(t=0.21, p=0.84)$. There was also no statistically significant relationship between the size of schools and their consent rate $(\mathrm{F}=0.08, \mathrm{p}=0.77)$.

In Northern Ireland, academic selection at 10-11 years of age is used to determine entrance to post-primary schools, with grammar schools selecting the higher performing children. Parental consent rates were similar between grammar and nongrammar schools: 63\% (range 46-80\%) in grammar schools and $66.5 \%$ (range $47-89 \%$ ) in non-grammar schools. $43.4 \%$ of postprimary school participants attend grammar school.

Table 2 summarises the characteristics of the participants.

\section{DISCUSSION}

The participation rate in the NICER study compares favourably with the Collaborative Longitudinal Evaluation of Ethnicity and Refractive Error study, a multicentre study of refractive error in childhood in the USA where consent rates ranged from $30 \%$ to $50 \% .{ }^{17}$ However, despite using similar procedures and protocols, the parental consent rate was lower for the NICER study than the Sydney Myopia Study (75\% in 12-year-olds, 79\% in 6-yearolds), ${ }^{27}$ a large-scale epidemiological survey of refractive error in Australian schoolchildren.

Because of ethical constraints, it is difficult to establish reasons for lack of parental consent in the NICER study. Differing cultural attitudes to research may help explain the variation in participation rates between similar studies in other countries. Lack of consent because of non-return of the forms was greater in post-primary school children, possibly because it was easier for primary school teachers to monitor and encourage return of the forms. The variation in parental consent rate between schools reflected the variation in enthusiasm between teaching staff within the schools involved, with schools where one or more members of staff took an active interest in the study having the highest participation rates. A greater proportion of parents of primary school children actively declined consent; this may have been due to concerns about the use of eye drops.

Although it is not possible to establish if the consent rate affected the validity of the results, the schools' participation rates were not statistically significantly affected by the size of the school or the deprivation or population density of the area in which the school resides (figures 1 and 2). The ethnicity, sex distribution and type of schooling of participants are also comparable with those of the target population, supporting the assumption that the results are applicable to the general population. The proportion of post-primary schoolchildren in the sample who attend a grammar school is similar to the figure for Northern Ireland as a whole (43\% vs $42 \%$, respectively) (http://

Table 2 Characteristics of participants

\begin{tabular}{lll}
\hline & \multicolumn{2}{l}{ Age group } \\
\cline { 2 - 3 } & 6-7-year-olds & 12-13-year-olds \\
\hline \% White & 98.5 & 98.8 \\
\% Male* & 49.5 & 50.5 \\
Age range (years) $^{*}$ & $6.33-7.83$ & $12.08-14.08$ \\
Mean age (SD) & $7.07(0.38)$ & $13.1(0.38)$ \\
\hline
\end{tabular}

*Data from white participants only. 
Figure 1 Bar chart illustrating the consent rate from primary schools in deprived versus non-deprived areas and rural versus urban areas. 'Deprived' multiple deprivation measure (MDM) $\geq 20$; 'non-deprived' MDM $<20$. 'Urban' population density $\geq 10$ persons per hectare; 'rural' population density $<10$ persons per hectare.

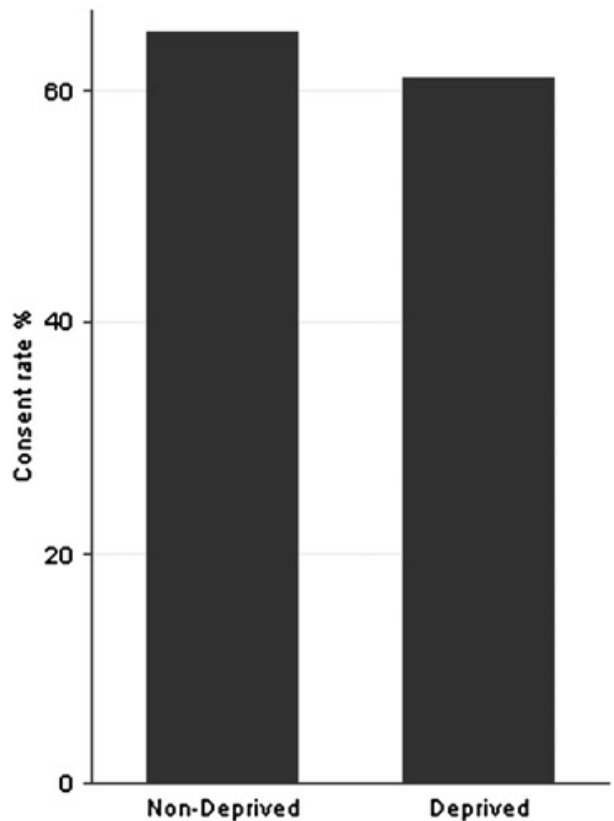

www.deni.gov.uk/). The gender of the participants closely matches that of the Northern Irish population (49\% male in Northern Ireland) (http://www.nisra.gov.uk/). Ethnicity was assessed by the study coordinator and confirmed by the response given in the parental questionnaire; this confirmation could not be carried out in cases of non-return of the questionnaire. Where the appropriate data are available, the ethnicity as identified by the study coordinator agreed with the parental identification of ethnicity in all cases, confirming the validity of assessing ethnicity in this manner in this study group. 98.8\% of school children in Northern Ireland are from a white ethnic group (http://www.nisra.gov.uk/), this figure closely matching the ethnicity of the participants.
Data from the NICER study will allow us to describe the prevalence of refractive error and to explore issues such as how lifestyle, refractive status and biometry measures influence refractive outcome. The influence of familial factors such as socioeconomic status, parental education and birth order on visual development during childhood will also be investigated.

The data will also enable us to quantify the extent to which uncorrected refractive error is a problem in this UK population and will provide evidence of the effectiveness of the Northern Ireland vision screening program in identifying uncorrected refractive error and reduced vision in childhood.

Future plans include the use of the sampling frame to examine trends and patterns in the prevalence and development of
Figure 2 Bar chart illustrating the consent rate from post-primary schools in deprived versus non-deprived areas and rural versus urban areas. 'Deprived' multiple deprivation measure (MDM) $\geq 20$; 'non-deprived' MDM $<20$. 'Urban' population density $\geq 10$ persons per hectare; 'rural' population density $<10$ persons per hectare.
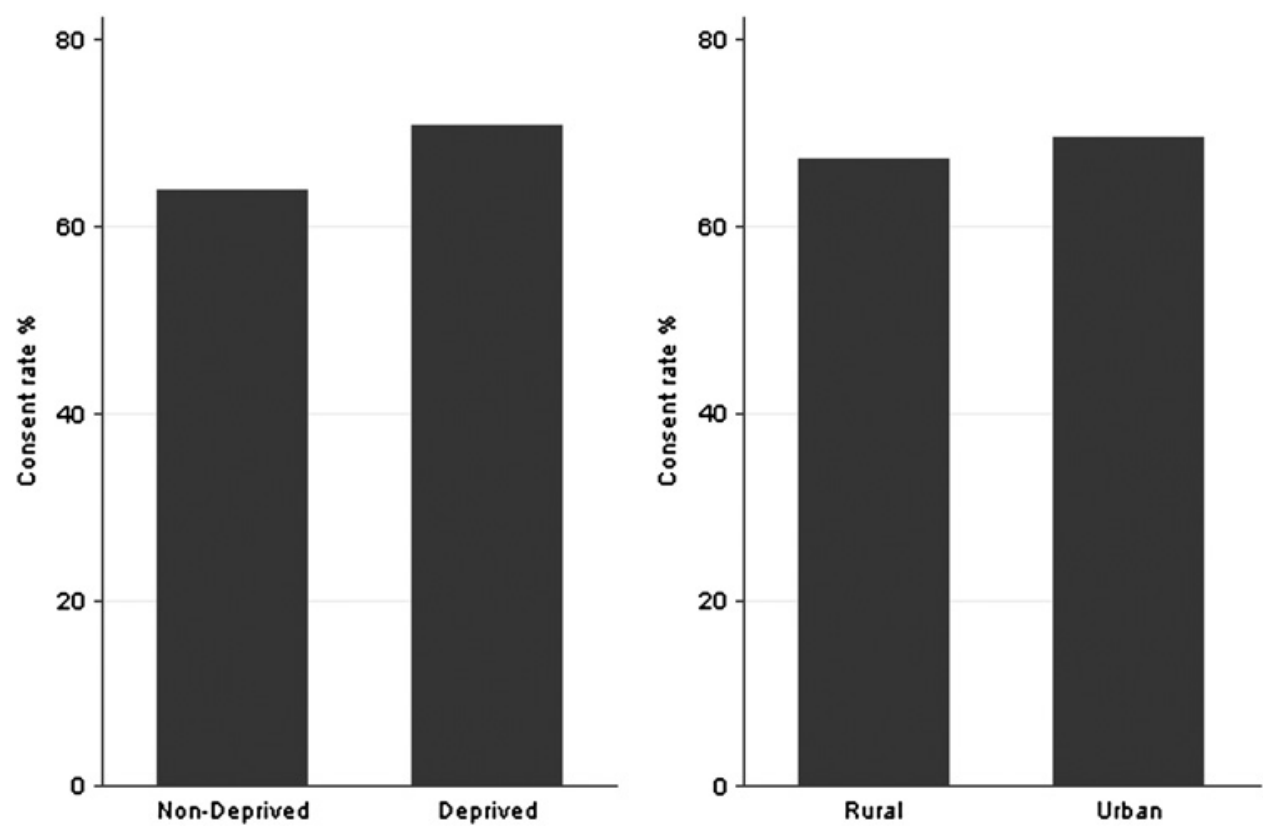
refractive status in childhood. Participants in the current study are currently being re-examined 3 years after they originally participated in the NICER study, providing useful longitudinal data on refractive error and its correlates.

Data from this population will provide a valuable database on refractive error distribution in a white population. Comparisons with data from white participants in the multiethnic Aston Eye Study will establish the generalisability of the findings to the UK population as a whole. The outcomes of these sister studies will be used to examine the influence of ethnicity on childhood refractive error in the UK.

\section{CONCLUSIONS}

This report describes the protocol used by the NICER study. The use of cycloplegic autorefraction, considered the gold standard for epidemiological studies of refractive error, will facilitate comparisons with other such studies, including the Sydney Myopia Study ${ }^{18}$ and the Refractive Error Study in Children. ${ }^{1}$

With participation rates of $57 \%$ (6-7-year-old children) and $60 \%$ (12-13-year-old children), the required sample size was achieved for both age groups. Most of the subjects in both age groups participated fully with data collection. The sex distribution and ethnicity of participants closely match the Northern Irish child population, supporting the robustness of the data.

Future papers will describe the prevalence of myopia, hyperopia and astigmatism within this population. Collaborative reports from the NICER study and the Aston Eye Study will compare the distribution of SER and ocular biometric parameters in schoolchildren in Northern Ireland with those of their multiracial peers in England.

Acknowledgements The authors are grateful to the College of Optometrists who provided a research scholarship to Lisa O'Donoghue, to the Northern Ireland Optometric Society who provided funding for the portable digital fundus camera and to Prof Peter Whincup for support in the design of the questionnaires.

Funding College of Optometrists, London, UK; Northern Ireland Optometric Society, Dromore, Co. Down,UK

\section{Competing interests None.}

Ethics approval This study was conducted with the approval of the University of Ulster Research Ethics Committee.

Provenance and peer review Not commissioned; externally peer reviewed.

\section{REFERENCES}

1. Negrel AD, Maul E, Pokharel GP, et al. Refractive error study in children: sampling and measurement methods for a multi-country survey. Am J Ophthalmol 2000:129:421-6.

2. Rose K, Smith W, Morgan I, et al. The increasing prevalence of myopia: implications for Australia. Clin Experiment Ophthalmol 2001;29:116-20.

3. Ip JM, Robaei D, Kifley A, et al. Prevalence of hyperopia and associations with eye findings in 6- and 12-year-olds. Ophthalmology 2008;115:678-85.e1.
4. Saw SM, Gazzard G, Shih-Yen EC, et al. Myopia and associated pathological complications. Ophthalmic Physiol Opt 2005;25:381-91.

5. McCarty CA, Taylor HR. Myopia and vision 2020. Am J Ophthalmol 2000:129:525-7.

6. Dayan YB, Levin A, Morad Y, et al. The changing prevalence of myopia in young adults: a 13-year series of population-based prevalence surveys. Invest Ophthalmol Vis Sci 2005;46:2760-5.

7. Saw SM. A synopsis of the prevalence rates and environmental risk factors for myopia. Clin Exp Optom 2003;86:289-94.

8. Junghans BM, Crewther SG. Prevalence of myopia among primary school children in eastern Sydney. Clin Exp Optom 2003;86:339-45.

9. Saw SM, Goh PP, Cheng A, et al. Ethnicity-specific prevalence rates of refractive errors vary in Asian children in neighboring Malaysia and Singapore. Br J Ophthalmol 2006;90:1230-5

10. Donnelly UM, Stewart NM, Hollinger M. Prevalence and outcomes of childhood visual disorders. Ophthalmic Epidemiol 2005;12:243-50.

11. Pointer JS. A 6-year longitudinal optometric study of the refractive trend in schoolaged children. Ophthalmic Physiol Opt 2001;21:361-7.

12. Rudnicka AR, Owen CG, Richards M, et al. Effect of breastfeeding and sociodemographic factors on visual outcome in childhood and adolescence. Am J Clin Nutr 2008;87:1392-9.

13. Sorsby A, Benjamin B, Sheridan M, et al. Refraction and its components during the growth of the eye from the age of three. Memo Med Res Counc 1961;301:1-67.

14. Williams C, Miller LL, Gazzard G, et al. A comparison of measures of reading and intelligence as risk factors for the development of myopia in a UK cohort of children. Br J Ophthalmol 2008;92:1117-21.

15. Williams C, Northstone K, Howard M, et al. Prevalence and risk factors for common vision problems in children: data from the ALSPAC study. $\mathrm{Br} J \mathrm{~J}$ Ophthalmol 2008;92:959-64

16. Fotedar R, Rochtchina E, Morgan I, et al. Necessity of cycloplegia for assessing refractive error in 12-year-old children: a population-based study. Am J Ophthalmol 2007;144:307-9

17. Zadnik K, Manny RE, Yu JA, et al. Ocular component data in schoolchildren as a function of age and gender. Optom Vis Sci 2003;80:226-36.

18. Ojaimi E, Rose KA, Smith W, et al. Methods for a population-based study of myopia and other eye conditions in school children: the Sydney Myopia Study. Ophthalmic Epidemiol 2005;12:59-69.

19. He M, Huang W, Zheng $Y$, et al. Refractive error and visual impairment in school children in rural southern China. Ophthalmology 2007:114:374-82.

20. Pokharel GP, Negrel AD, Munoz SR, et al. Refractive error study in children: results from Mechi Zone, Nepal. Am J Ophthalmol 2000;129:436-44.

21. Logan NS, Rudnicka AR, Shah P, et al. The epidemiology of refractive error in UK children: the Aston Eye Study methodology. Invest Ophthalmol Vis Sci 2007;48:ARVO E-abstract 4847.

22. Logan NS, Rudnicka AR, Shah P, et al. Ethnic differences in ocular refraction and biometry in UK children: evidence from the Aston Eye Study. Invest Ophthalmol Vis Sci 2008;49:ARVO E-abstract 2602.

23. Santodomingo-Rubido J, Mallen EA, Gilmartin B, et al. A new non-contact optical device for ocular biometry. Br J Ophthalmol 2002;86:458-62.

24. Chat SW, Edwards MH. Clinical evaluation of the Shin-Nippon SRW-5000 autorefractor in children. Ophthalmic Physiol Opt 2001;21:87-100.

25. Carkeet A, Saw S, Gazzard G, et al. Repeatability of IOLMaster biometry in children. Optom Vis Sci 2004:81:829-34.

26. Gilbert CE, Ellwein LB. Refractive error study in children study group prevalence and causes of functional low vision in school-age children: results from standardized population surveys in Asia, Africa, and Latin America. Invest Ophthalmol Vis Sci 2008;49:877-991.

27. Robaei D, Huynh SC, Kifley A, et al. Correctable and non-correctable visual impairment in a population-based sample of 12-year-old Australian children. Am J Ophthalmol 2006;142:112-8.

28. Robaei D, Rose K, Ojaimi E, et al. Visual acuity and the causes of visual loss in a population-based sample of 6-year-old Australian children. Ophthalmology 2005;112:1275-82. 